\title{
RESUMEN
}

La importancia que tuvo la inmigración europea en el periodo de la conformación de la sociedad regional no indígena de la Araucanía a fines del siglo XIX y principios del XX se manifiesta de manera significativa en la esfera educativa. En efecto, si bien demográficamente la presencia de inmigrantes europeos nunca fue mayoritaria, sí dejó sus huellas en el importante rol que desempeñaron las comunidades de inmigrantes en el surgimiento y desarrollo de escuelas privadas, en particular, alemanes, franceses, suizos e ingleses. Muchos de estos inmigrantes eran protestantes y anglicanos, hecho que sin duda incidió en la creación de escuelas no católicas, en un contexto en que las órdenes misioneras franciscana y capuchina tenían a su cargo gran parte de la educación de la región. En este artículo presentamos los antecedentes, surgidos de una investigación de antropología histórica, sobre los flujos de inmigración europea que avalan las afirmaciones anteriores.

Palabras claves:

Colonos Araucanía - Inmigrantes europeos Araucanía - Educación Privada Araucanía Escuelas Francesas Araucanía - Colegios Alemanes Araucanía - Colegios protestantes Araucanía.

\begin{abstract}
The importance that had the European immigration in the period of the conformation of the regional society not indigenous in the Araucanian region at the end of the $19^{\text {th }}$ century and the beginning of the $20^{\text {th }}$ century demonstrate in a significant way in the educational sphere. Indeed, though demographically the presence of European immigrants was never majority population they played an important role in the emergence and development of private schools, especially Germans, Frenchmen, Swiss and English. Many of these immigrants were Protestants and Anglicans, a fact that undoubtedly affected in the creation of not catholic schools because the Franciscan and Capuchin missionaries had a great control on the educational system. In this article show data originated of an historical anthropological research on the flows of European immigration that supports the previous arguments.
\end{abstract}

Key words:

Araucanian Colonists - Araucanian European Immigrants - Araucanian Private Education Araucanian French's School - Araucanian Germans School - Araucanian Protestants School. 


\begin{abstract}
Los colonos y la escuela en la Araucanía: Los inmigrantes europeos y el surgimiento de la educación privada Laica y Protestante en la Región de la Araucanía (1887-1915) José Manuel Zavala Cepeda

Pp. 268 a 286

\section{LOS COLONOS Y LA ESCUELA EN LA ARAUCANÍA: LOS INMIGRANTES EUROPEOS Y EL SURGIMIENTO DE LA EDUCACIÓN PRIVADA LAICA Y PROTESTANTE EN LA REGIÓN DE LA ARAUCANÍA $(1887-1915)^{1}$}

\author{
José Manuel Zavala Cepeda $\left(^{*}\right)$
}

\title{
INTRODUCCIÓN
}

Es, sin duda, en el terreno educativo donde se puede apreciar con mayor claridad la huella de los procesos que se conjugaron en la génesis de la sociedad regional no indígena de la Araucanía, y que fueron fortaleciendo o haciendo desaparecer las diferencias culturales, lingüísticas y religiosas de los inmigrantes europeos. Aun hoy día es posible percibir, aunque de manera desdibujada, la dinámica que se puso en marcha, las influencias y las particularidades que los estilos, valores y prácticas ejercidos en las escuelas plasmaron en la formación de varias generaciones de hombres y mujeres de esta región.

Desarrollaremos a continuación, en primer lugar, los antecedentes respecto de la importancia de las comunidades de inmigrantes europeos al momento de la consolidación de la sociedad regional no indígena y del surgimiento de la escuela;

\footnotetext{
(*) Doctor en Antropología por la Universidad de París III, Sorbonne Nouvelle. Docente-Investigador de la Escuela de Antropología de la Universidad Católica de Temuco.

Artículo recibido el 16 de enero de 2008. Aceptado por el Comité Editorial el 14 de marzo de 2008.

Correo electrónico: zavalacepeda@gmail.com

1 Este artículo es fruto del proyecto DIUCT N² 2003-1-02 “Flujos Migratorios, Identidad Cultural y Estructura Social de la Región de la Araucanía: Análisis del impacto sociocultural de los movimientos demográficos en la IX Región desde la Antropología Histórica" financiado por la UC Temuco, a través de su Dirección General de Investigación. Nuestros más sinceros agradecimientos a la estudiante de Licenciatura en Antropología Angélica Lezano por su importante colaboración en el desarrollo de esta investigación.
} 
luego presentaremos los proyectos educativos en que dichas comunidades participaron para finalmente referirnos a algunos proyectos educativos posteriores de origen protestante.

\section{LOS INMIGRANTES EUROPEOS Y LA CUESTIÓN EDUCATIVA EN LOS INICIOS DE LA SOCIEDAD REGIONAL NO INDÍGENA}

\subsection{Importancia de la inmigración europea en la Araucanía}

Entre fines de 1883 y principios de 1884 se instalaron en la parte norte de la región de la Araucanía 500 familias de españoles, franceses, italianos, suizos y alemanes en las colonias inmediatas a Victoria, Quechereguas, Huequen, Traiguén y Contulmo ${ }^{2}$. Fue el inicio de un ciclo inmigratorio europeo importante hacia las áreas rurales de la región, promocionado y planificado por el Estado, que tuvo su momento de auge entre 1883 y 1890, cuando llegaron en total 6880 inmigrantes europeos en calidad de colonos ${ }^{3}$.

Si sumamos el número de inmigrantes registrados para el período 1883-1890, 6880 colonos, a las estimaciones que hemos podido hacer sobre la base de los registros de las colonias rurales de la Araucanía para el decenio inmediatamente posterior (1891-1900), 500 colonos, y para los últimos doce años de este ciclo inmigratorio masivo (1901-1912), 1750 colonos, podemos obtener una cifra general aproximada de 9130 europeos llegados como colonos a las áreas rurales de la Araucanía entre 1883 y 1912, cifra sin duda muy aproximada y que no considera a los otros inmigrantes europeos arribados por sus propios medios y sin la condición de colono a la región. Con posterioridad a 1912, la llegada de inmigrantes europeos que pudieran optar al título de colono y asegurarse así el acceso a una propiedad rural disminuyó considerablemente; en algunos casos, antiguos territorios de colonización que no habían logrado consolidarse completamente recibieron algunas nuevas familias de inmigrantes europeos, como ocurrió en el caso de Pichi-Chelle, en el área de colonización del Budi, donde entre 1924 y 1928 llegaron cuatro familias alemanas católicas ${ }^{4}$.

A este flujo inmigratorio "registrado" gracias a las estadísticas de las colonias rurales habría que agregar todos los demás inmigrantes que al no establecerse en dichos enclaves quedaron fuera de los registros y cuyo número es muy difícil de estimar. En la mayoría de los casos, esta inmigración espontánea estuvo estrechamente asociada al desarrollo de los nacientes centros urbanos que comenzaban a articular los sistemas de distribución, servicios, transporte e industria de la región, logrando a veces constituir pequeños núcleos de nacionalidades gracias a redes familiares o

\footnotetext{
2 Memoria Anual del Ministerio de Relaciones Exteriores (M.RREE), 1884: 135-158.

3 El marco jurídico fundamental que regula los procesos de colonización de la Araucanía con inmigrantes europeos está dado por las leyes de colonización del 4 de diciembre de 1866 y del 4 de agosto de 1874 . Los territorios destinados a colonización fueron subdivididos en hijuelas, propiedades agrícolas de entre 100 a 300 hectáreas aproximadamente, y entregados gratuitamente a quienes obtenían la calidad de "colono" bajo ciertas condiciones. 4 Se trata de 4 familias compuestas por 22 personas (Cf. Entrevista a don Josef Hund).
} 
comunitarias alimentadas en una o más generaciones, como fue el caso de un núcleo judío sefaradí procedente de Monastir, Macedonia ${ }^{5}$, instalado en Temuco, y de diversas comunidades árabes ${ }^{6}$.

\subsection{Los colonos y el "problema" educativo}

Los primeros inmigrantes europeos que se instalaron en las colonias rurales de la Araucanía debieron resolver cuestiones de índole económica, social y cultural inevitables de abordar en todo proyecto colonizador. Por una parte, requerían crear las bases logísticas necesarias para la habitabilidad y la explotación agrícola de sus hijuelas; por otra parte, debían construir los soportes de una vida social que tuviera "sentido" a partir de sus propios referentes culturales. En este último aspecto, era vital crear los espacios de encuentro y de conservación de la "identidad" y de la "vida comunitaria" previa, pero también aquellos que pudieran plasmar un proyecto de futuro, en particular para los hijos.

Es así como muy tempranamente surgieron las inquietudes e iniciativas con respecto a la construcción de iglesias y escuelas, dos espacios importantes cuando se trata de identidad, vida comunitaria y proyección de futuro. En efecto, tanto el lugar de culto como el de la educación de los hijos aparecen como una preocupación importante entre los colonos, y muchas veces fueron planteados como dos aspectos de una misma problemática; nada de sorprendente si se tiene en cuenta el importante rol educativo que han jugado y juegan las iglesias y la estrecha relación existente entre la formación religiosa y los primeros años de escolarización.

Hay que considerar que núcleos de familias de una misma nacionalidad y, en muchos casos, de una misma religión se encontraban relativamente aislados, pero eran lo suficientemente numerosos como para pensar en un proyecto común. En general, se trataba de familias jóvenes con un número significativo de hijos menores por educar ${ }^{7}$

En ese contexto, la pertenencia religiosa emerge como un factor importante a la hora de enfrentar el tema de la educación de los hijos, en particular para las familias protestantes pues hasta ese momento eran las órdenes franciscana y capuchina, las organizaciones religiosas con mayor presencia educativa en las áreas de colonización, que a su vez eran las áreas de evangelización y escolarización de una población mapuche que estaba siendo arrinconada o desalojada de ellas en provecho del proyecto colonizador. Cabe señalar que, aunque no hemos encontrado datos generales que expliciten la pertenencia religiosa de los inmigrantes, es posible inferir a base de diversas evidencias que una buena parte de los alemanes y muchos suizos de lengua alemana eran protestantes, así como los ingleses eran sin duda anglicanos.

\footnotetext{
5 En 1916 residen en Temuco alrededor de 25 familias provenientes de la ciudad de Bitolj (antigua Monastir), Macedonia; en 1928 se inaugurará en Temuco, un colegio Hebreo que funcionará hasta mediados del siglo XX (Cohen, 2002: 48).

6 En 1920 la población árabe de la Araucanía era de 469 personas y en 1930 de 445, concentrándose su mayor número en Temuco (Rebolledo, 1991: 78-79). Las nacionalidades presentes en 1920 por orden de importancia son: Saudita (207), Siria (104), Palestina (91), Turca (66) y Egipcia (1).

7 El promedio de miembros de las familias de colonos es en 1890 de cinco personas (M. RREE, 1890: 490).
} 
Por otra parte, la cuestión lingüística figura igualmente entre los inmigrantes como un factor determinante a la hora de reflexionar sobre un proyecto educativo para sus hijos. En efecto, recordemos que gran parte de los inmigrantes provenían de países donde la lengua es una marca de reconocimiento identitario nacional, situado esto en un contexto histórico de expansión colonial, la dimensión lingüística adquiere una importancia aún más considerable y se asocia a un determinado proyecto civilizatorio. Esto puede vislumbrarse, por ejemplo, en la clásica rivalidad franco-germana, característica de fines del siglo XIX y principios del siglo XX. El manejo de una determinada lengua se vuelve entonces un criterio trascendente a la hora de identificarse con una nacionalidad de origen o con un determinado proyecto civilizatorio asociado a dicha nacionalidad.

Los dos factores anteriormente señalados, el religioso y el lingüístico, asociados a una realidad de casi inexistencia de infraestructura y servicios educativos previos, incidieron sin duda en la germinación temprana de proyectos educativos autogestionados por los propios colonos en las áreas próximas a sus lugares de residencia.

\subsection{La escuela al momento de la llegada de los primeros colonos}

Cuando se inicia el ciclo de inmigración colona en la Araucanía, en 1883, la labor educativa estaba en la mayor parte del territorio en manos de los misioneros franciscanos y capuchinos, quienes realizaban tareas de evangelización entre los mapuches; sin embargo, hubo igualmente una temprana presencia de la escuela pública en el área norte y noroeste de la región, en las zonas de ocupación y concentración de la población chilena, particularmente en Angol, Collipulli y Purén, como lo señala el estudio de Sol Serrano ${ }^{8}$.

En las misiones franciscanas y capuchinas, de manera complementaria a la acción evangelizadora y como una forma de llevarla a cabo, se desarrollaban tareas de alfabetización y de primeras letras destinadas a los niños indígenas, en las cuales también participaban los hijos de chilenos e inmigrantes instalados en esos territorios. La acción evangelizadora formaba parte de una estrategia política mayor de subordinación de la población mapuche al Estado nacional chileno y de aculturación al mundo cristiano-occidental, por lo que se solicitaba insistentemente a los misioneros que asumieran tareas educativas e hicieran funcionar escuelas en sus casas misionales, comprometiéndose el Estado al financiamiento parcial de dicha labor ${ }^{9}$.

Durante la segunda mitad del siglo XIX, podemos distinguir dos sectores con presencia misionera católica en la Araucanía:

a) La parte norte de la Araucanía y la costa de Arauco, donde los recoletos

8 En 1858 había 22 escuelas públicas (20 fiscales y dos municipales) en la Provincia de Arauco: 12 se situaban en el Departamento de Laja, seis en el de Nacimiento y cuatro en el de Arauco (Sol Serrano, 1995-96: 453). Hacia 1879 en el Territorio de Colonización de Angol había 15 escuelas (cinco fiscales, ocho privadas y dos misionales): seis se situaban en el pueblo de Angol, cinco en Collipulli y las restantes en Purén, Tijeral y Lumaco (Sol Serrano, 1995-96: 457).

9 Sol Serrano, 1995-96: 435. 
franciscanos ${ }^{10}$ pertenecientes a los colegios de Chillán y Castro realizaban tareas de evangelización. En 1859, se señalan misiones franciscanas en Nacimiento, Tucapel, Rosales y Malvín ${ }^{11}$. Hacia 1888 existían misiones franciscanas en Angol, Nacimiento, Mulchén, Collipulli, Traiguén, Lumaco, Chol-Chol, Tucapel de Cañete y Tirúa ${ }^{12}$. En algunas de ellas funcionaban escuelas a las que asistían los niños mapuches y, con toda seguridad, los hijos de las familias chilenas y extranjeras residentes. En particular en Angol se da cuenta de la existencia de un internado al que asistían entre 20 y 30 niños indígenas ${ }^{13}$.

b) La parte sur de la Araucanía y norte de la provincia de Valdivia, donde se instalaron a partir de 1848 capuchinos italianos, principalmente en la costa, al sur del río Imperial, en San José de la Mariquina (1850), Imperial Bajo (1850, actual Puerto Saavedra), Queule (1854-55) y Toltén (1861). Todas estas misiones poseían escuelas hacia $1865^{14}$. Dichos capuchinos tenían además a su cargo todas las misiones situadas en el centro y sur de la provincia de Valdivia. Posteriormente, a partir de 1896, los capuchinos italianos fueron reemplazados por capuchinos bávaros, quienes continuaron y ampliaron la labor misional hacia el interior de la parte norte de la provincia de Valdivia.

No existe entonces, a la llegada de los primeros inmigrantes colonos, un sistema educativo consolidado en los territorios destinados a la colonización aunque sí están presentes algunas escuelas misionales católicas fundadas en las áreas en que se concentraba la población mapuche y algunas escuelas públicas en los centros poblados de la parte norte y noroeste de la región.

\section{LOS PROYECTOS EDUCATIVOS DE LOS COLONOS}

Entre 1887 y 1915 se crearon en la Araucanía al menos diecinueve establecimientos educacionales relacionados con las comunidades de inmigrantes europeos asentados en la región. Doce de estos proyectos estuvieron relacionados con la comunidad alemana, cinco con la comunidad francesa, uno con la comunidad suiza y uno con la comunidad inglesa; en los últimos dos casos, se trató de proyectos originados en misiones protestantes, la del pastor Leutwyler y la de los anglicanos de la SAM (South American Missionary Society) respectivamente. En el caso de los doce establecimientos alemanes, al menos dos pueden ser identificados claramente como escuelas protestantes, se trata de las escuelas "evangélicas" de El Salto (1894) y de Lautaro (1899).

${ }^{10}$ La importancia de los franciscanos en la educación se aprecia en la existencia actual de colegios de la orden de San Francisco en las principales ciudades de la Araucanía. Entre los más antiguos, figuran el de Temuco (1885), el de Angol (1889) y el de Lautaro (1895) (Información de la Fundación Educacional de la Conferencia Episcopal de Chile, 2006).

${ }_{11}$ Sol Serrano, 1995-96: 447-448.

12 M. RREE, 1888: 57-61.

${ }_{13}$ M. RREE, 1888: 57- 61, 103 .

${ }^{14}$ Sol Serrano, 1995-96: 442. 
Tabla $\mathbf{N}^{\circ}$ 1. Escuelas ligadas a comunidades de inmigrantes, creadas entre 1887 y 1915

\begin{tabular}{|l|l|l|}
\hline Fecha Fundación & Lugar & Comunidad \\
\hline 1887 & Temuco & Alemana \\
\hline 1892 & Traiguén & Francesa \\
\hline 1892 & Ercilla & Francesa \\
\hline 1893 & Victoria & Francesa \\
\hline 1893 & Lautaro & Francesa \\
\hline 1893 & Traiguén & Suiza \\
\hline 1893 & Contulmo & Alemana \\
\hline 1894 & El Salto & Alemana \\
\hline 1894 & Nueva Imperial & Alemana \\
\hline 1895 & Purén & Alemana \\
\hline 1895 & Traiguén & Alemana \\
\hline 1898 & Victoria & Alemana \\
\hline 1899 & Lautaro & Alemana \\
\hline 1900 & Ercilla & Alemana \\
\hline 1904 & Quillem & Alemana \\
\hline 1904 & Galvarino & Francesa \\
\hline 1906 & Gorbea & Alemana \\
\hline 1910 & Faja Maisan & Alemana \\
\hline $1910-1915$ & Temuco & Inglesa \\
\hline
\end{tabular}

Fuentes: Frey, 1910; BAF 1884-1906; S. Venezian, 1993; B. Bazley [1999]; A. Dufey, 2000.

La mayoría de estos establecimientos fueron el fruto de proyectos educativos autogestionados por los propios colonos con el apoyo de las iglesias y/o países de origen. Se trata, en sus inicios, de escuelitas primarias situadas en las proximidades de las colonias que agrupaban las propiedades de los inmigrantes donde muy tempranamente se organizaron comités o cooperativas locales por nacionalidad para resolver el problema de la educación de los hijos; en particular, este fue el caso de los colonos alemanes y franceses, que optaron por organizarse sobre la base de sus respectivas nacionalidades de origen.

Estas escuelas de colonos, a diferencia de otros proyectos educativos posteriores de origen protestante, fueron creadas principalmente para la formación de los hijos de inmigrantes e implementaron una educación lingüística y culturalmente muy asociada a sus países de origen, de donde provenían igualmente la mayor parte de los maestros. Estas escuelas se presentaron más bien como proyectos laicos o interconfesionales de enseñanza elemental.

\subsection{Las escuelas de la Alianza Francesa}

Los inmigrantes franceses llevaron a cabo una importante iniciativa en materia educativa en la región en torno a los núcleos de colonos franceses y suizos francófonos. 
Entre 1892 y 1904 se crearon cinco escuelas en las áreas de colonización de la parte norte de la región: en Traiguén (1892), Ercilla (1892), Victoria (1893), Lautaro (1893) y Galvarino (1904). En el caso de Traiguén, el establecimiento educacional que hoy lleva el nombre de liceo Luis Pasteur se mantuvo hasta mediados del siglo XX como un establecimiento ligado cultural, lingüística y administrativamente al mundo francés de tradición católica.

Un rol central en el desarrollo de las escuelas francesas en la región, lo jugó la Alianza Francesa, asociación sin fines de lucro, creada en París en 1883, para difundir la lengua y la cultura francesas en el mundo en un contexto de auge de los proyectos coloniales de las potencias europeas, cuando cada cual trataba de implementar acciones tendientes a garantizar su presencia en los diversos continentes.

La Alianza Francesa se organizó en torno a una red de comités locales presente en diversos lugares del planeta que recibían apoyo y se articulaban a partir de la base de la organización situada en París. Dichos comités locales constituían sociedades benéficas que se ocupaban de buscar los financiamientos y asegurar la gestión de las escuelas; se trataba de organizaciones laicas, pero cuyos miembros eran fundamentalmente de tradición católica.

La historia de las escuelas francesas de la Araucanía puede ser reconstituida recorriendo los boletines de la Alianza Francesa que se conservan aún en su sede central en París.

En primer lugar, cabe señalar la rapidez e importancia que adquirió la acción de la Alianza Francesa en Chile a pesar de no ser un país situado en la órbita colonial francesa. En efecto, ya en 1885, dos años después de su creación, se reconoce un Comité Regional de la Alianza en Valparaíso, principal ciudad de inmigración en Chile, y surgen rápidamente otros comités en distintos puntos de país.

En los boletines de la Alianza de 1891, se señala que Traiguén y Ercilla son centros de colonos franceses en el sur del país; se precisa igualmente, que la región de la Araucanía cuenta con más de 500 familias francesas y 2.000 niños "sin medios para educarse" ${ }^{\prime \prime 15}$.

En 1892, se abrieron las primeras escuelas de nombre Alianza Francesa en estos lugares; Ercilla contaba con 26 alumnos y Traiguén con 38 alumnos, aproximadamente; según el boletín de la Alianza, el aporte de los colonos fue fundamental para levantar las primeras escuelas ${ }^{16}$.

Hacia 1894, Chile contaba con ocho comités locales de la Alianza Francesa: Valparaíso, Santiago, Iquique, Ercilla, Traiguén, Quino, Lautaro y Victoria. Como puede apreciarse, cinco de los ocho comités chilenos se situaron en la región de la Araucanía, específicamente en los lugares donde se habían establecido los colonos franceses.

En 1904, la Alianza Francesa mantenía funcionando cinco escuelas en la región: Ercilla (1892), Traiguén (1892), Victoria (1893), Lautaro (1893) y Galvarino (1904) ${ }^{17}$.

\footnotetext{
15 B.A.F. $\mathrm{N}^{\circ}$ 38, 1891: 129.

16 B.A.F. $N^{\circ} 40,1892: 68$.

17 B.A.F, $\mathrm{N}^{\circ} 98,1904: 308$
} 
Aunque las escuelas francesas no estaban dirigidas exclusivamente a los hijos de franceses, su proyecto educativo era francés y, sin duda, entre las primeras generaciones de sus alumnos predominaron los franceses y los suizos francófonos.

En el caso de la escuela de Traiguén, por ejemplo, se dejaba en claro en sus estatutos que se admitirían "alumnos de ambos sexos, sin distinción de nacionalidad, estirpe o credo religioso", pero se precisaba igualmente que se enseñaría de "preferencia la lengua francesa"18.

En la práctica, los alumnos de origen francés y francosuizo fueron, al parecer, mayoritarios en los primeros decenios de vida de las escuelas francesas. Así, por ejemplo, en 1894 la escuela de Traiguén, creada dos años antes, contaba entre sus 79 alumnos a 47 franceses, 16 chilenos, 6 españoles, 4 ingleses, 4 suizos y 2 alemanes $^{19}$ y, en 1906, la escuela de Victoria, creada trece años antes, contaba entre sus 108 alumnos a 39 franceses, 40 suizos, 21 chilenos, 6 alemanes y 2 italianos ${ }^{20}$.

Se comprende esta predominancia de los descendientes de franceses y francosuizos, en la medida que una misión fundamental de estos proyectos educativos era garantizar la perennidad de la lengua y cultura francesas en estos verdaderos enclaves inmigratorios, donde muchos inmigrantes franceses, paradójicamente, parecían no manejar lo suficiente la lengua ni la cultura francesa oficiales, pues provenían de áreas culturales y lingüísticas diferenciadas como el País Vasco, el Bearn o los Alpes suizos y se encontraban en una situación de minoría. Las palabras de Monsieur Leconte, importante vecino francés de Traiguén, señalan esta preocupación, cuando dice:

"Los hijos, ignoran, tanto como sus padres, la verdadera lengua nacional, acostumbrados a hablar en patois o en chileno, habrían perdido toda noción del francés ${ }^{21}$.

La idea de una "escuela francesa" en medio de los campos de una Araucanía que seguramente representaba para los promotores del proyecto inmigratorio la antítesis de la cultura y la civilización, juega quizás un doble rol: uno de primer plano, relativo a difundir y mantener la cultura y la lengua francesas, y otro de segundo plano, consistente en "hacer aun más franceses" a quienes en realidad no lo eran tanto, pues provenían de regiones con culturas y lenguas que estaban viviendo procesos de subordinación a las lenguas y culturas centrales, en una época de consolidación de los estados naciones europeos, en que los candidatos a emigrar se encontraban con toda seguridad más fácilmente entre aquellos que vivían situaciones de desarraigo, persecución o marginación, que entre aquellos que estaban más a gusto con sus vidas. En este sentido, una labor educativa que entregara el "patrón" lingüístico y cultural ideal era concordante con el espíritu del proyecto inmigratorio.

Otro elemento que sin duda incidió en la concreción de los proyectos locales de educación franceses fue la preocupación existente en los medios que se agruparon

\footnotetext{
18 Revista Centenario, 1991: 3.

19 B.A.F. $N^{\circ}$ 51, 1894: 188.

20 B.A.F. No $105,1906: 310$.

21 B.A.F. $N^{\circ} 41,1892: 101-102$. "Les enfants, ignorant comme leurs parents d'allieurs de la vraie langue nationale, habitués á parler patois ou chilien, eussent bientôt perdu toute notion du francais" [traducción al castellano es nuestra].
} 
en torno a la Alianza Francesa por la expansión de la influencia alemana; así se vislumbra en un informe de 1893 sobre las actividades de la Alianza Francesa en Chile, donde se señala que "los comités del Sur toman cada vez más extensión y luchan contra la influencia alemana 22 ". Esta "rivalidad" franco-germana (no olvidemos que se trata de países enemigos durante gran parte de la segunda mitad del siglo XIX y primera mitad del siglo XX), parece haberse trasladado a la Araucanía y expresado, entre otros escenarios, en el educativo. Al parecer, los franceses veían con preocupación el desarrollo de colegios alemanes capaces de captar hijos de colonos de otras nacionalidades y chilenos. Pero no se trataba de una rivalidad únicamente de "nacionalidades" sino también de una diferencia religiosa, aunque más discreta, pues los proyectos educativos franceses estaban asociados al mundo católico en tanto los colegios alemanes aparecían más cercanos a los medios protestantes.

El proyecto educativo francés tuvo un rápido y amplio desarrollo en la enseñanza primaria, pero no perduró en la mayoría de los casos. La excepción fue la escuela de Traiguén, que se consolidó y extendió igualmente a la enseñanza secundaria asegurando la formación de los descendientes de colonos y de los jóvenes de la ciudad de Traiguén y sus alrededores, en un proyecto educativo claramente francés hasta mediados del siglo XX. Dicho establecimiento existe todavía con el nombre de Liceo Luis Pasteur, "Alianza Francesa" de Traiguén, pero en la actualidad se encuentra integrado al sistema educativo público chileno y no es reconocido como establecimiento de enseñanza francesa.

\subsection{Los Colegios Alemanes}

El proyecto educativo de los inmigrantes alemanes en Chile ha sido uno de los más sólidos y prolíferos entre las iniciativas surgidas de las comunidades inmigrantes. En el caso de la Araucanía, llama la atención la rápida organización de establecimientos educacionales en los principales núcleos urbanos y la continuidad de su actividad en el tiempo.

Los establecimientos alemanes se presentaron como iniciativas locales basadas en la organización de las comunidades alemanas (sociedades, cooperativas) y generalmente fueron de carácter laico aunque ligadas, muchas veces, a iglesias protestantes. Si bien no se definieron como proyectos reservados a la comunidad alemana, sin duda entre los alumnos de las primeras generaciones predominó la matriz cultural y lingüística alemana; por lo demás, la relevancia que le otorgaron las comunidades de inmigrantes alemanes a la escuela como medio de preservación y difusión de su lengua y cultura debe haber fortalecido la relación entre comunidad y escuela. Al respecto, son ilustrativas las palabras de Juan Frey, autor de una importante monografía de 1910 sobre el estado de los colegios alemanes de Chile, dice:

"Es de esperar que los niños de origen alemán conserven sus cualidades de raza $i$ de

${ }^{22}$ B.A.F. $\mathrm{N}^{\circ} 45,1893: 181$. “ Les Comités du Sud prennent de plus en plus d'éxtension et luttent contre l'influence allemande" [traducción al castellano es nuestra]. 
lengua i contribuyan a la vez a trasmitir a los demás las ventajas de la cultura i educación alemana ${ }^{23}$."

La monografía de Frey nos permite conocer el estado de los colegios alemanes de la Araucanía en 1909, los cuales sumaban en total 12 establecimientos (si incluimos a la localidad de Contulmo, que aunque situada actualmente en la Región del BíoBío, forma parte del mismo proceso histórico de colonización de la Araucanía) y contaban en total 354 alumnos. La tabla $n^{\circ} 2$ presenta el detalle del estado de los colegios alemanes de la Araucanía en 1909.

Tabla N ${ }^{\circ}$ 2. Los Colegios Alemanes en 1909

\begin{tabular}{|l|l|l|l|l|}
\hline Pueblo & Hombres & Mujeres & Total & Fundación \\
\hline Temuco & 35 & 36 & 71 & 1887 \\
\hline Contulmo & 30 & 24 & 54 & 1893 \\
\hline El Salto & & & & 1894 \\
\hline Nva. Imperial & 18 & 23 & 41 & 1894 \\
\hline Purén & 14 & 18 & 32 & 1895 \\
\hline Traiguén & 23 & 19 & 42 & 1895 \\
\hline Victoria & 21 & 7 & 28 & 1898 \\
\hline Lautaro & 12 & 8 & 20 & 1899 \\
\hline Quillem & 14 & 14 & 28 & 1904 \\
\hline Gorbea & $\ldots$ & $\ldots$ & 22 & 1906 \\
\hline Quilaco-Pucón & $\ldots$ & $\ldots$ & $\ldots$ & 1909 \\
\hline Ercilla & 10 & 6 & 16 & 1900 \\
\hline Total & $\mathbf{1 7 7}$ & $\mathbf{1 5 5}$ & $\mathbf{3 3 2 + 2 2}$ & \\
\hline \multicolumn{5}{|l}{ Fuente: Juan Frey, 1910.} \\
\end{tabular}

Se puede apreciar en esta tabla que de un total de 332 alumnos en que se identifica el género para el año 1909, un 46,7\% eran mujeres, es decir 155 alumnas, lo que sin duda representa para la época, un nivel importante de escolarización femenina entre la comunidad alemana. Podemos suponer que el hecho de que las niñas de origen alemán tuvieran la posibilidad de asistir a las escuelas alemanas, debe haber incidido en la capacidad que ha mostrado el alemán para mantenerse como lengua de comunicación familiar, en la medida que se les entregó a las mujeres la posibilidad de perfeccionarse en el aprendizaje y práctica de esta lengua.

El interés por contar con una educación alemana estuvo presente desde los inicios entre los colonos de los diversos lugares de la región y fue determinante en la creación de escuelas rurales, pero en algunos casos debieron esperar varios años. Así por ejemplo, los alemanes de la colonia de Quillen, una de las primeras fundadas con anterioridad a 1890, sólo contaron con su propia escuela en 1904, pero desde la fundación de colegios de lengua alemana y de orientación protestante o laica en la región se esforzaron por

${ }^{23}$ Frey, 1910: 357. 
enviar a sus hijos allí, teniendo como principales alternativas el Hogar Suizo la Providencia de Traiguén y los colegios alemanes de Victoria y Temuco ${ }^{24}$.

Diferente fue el caso de los alemanes brandeburgueses de la colonia El Salto, quienes ya en 1894 lograban levantar una escuela aunque solo eran 9 familias ${ }^{25}$; sin duda, incidió en este caso una estrecha relación entre una comunidad religiosa protestante constituida por las familias colonas y un proyecto educativo local, lo que se trasluce en el nombre del establecimiento: "Escuela Elemental Evangélica". En este caso, la escuela debe de haber sido el punto de reunión de la vida comunitaria, además del lugar de los servicios religiosos.

Cada colegio alemán era sostenido por una sociedad cooperativa compuesta por colonos del sector respectivo; en 1909, el número de socios iba de 12 en Lautaro a 48 en $\mathrm{TemuCO}^{26}$. La mayoría de los colegios alemanes eran escuelas elementales laicas o interconfesionales de orientación protestante a excepción de las escuelas de El Salto y de Lautaro, que se definían más específicamente como escuelas "evangélicas".

Entre los alumnos que asistían a los colegios alemanes a principios del siglo XX, se evidencia una diversidad de nacionalidades, credos y lenguas, pero con una cierta predominancia de la religión protestante y de la lengua alemana.

Así por ejemplo, en el caso del colegio alemán de Traiguén, en el mes de noviembre de $1909^{27}$ de un total de cuarenta y dos alumnos, dieciocho eran designados como alemanes, trece como chilenos, dos como italianos, tres como rusos y seis como suizos. $Y$ con respecto a la religión de estos mismos alumnos, se estimaba que veinte eran protestantes, quince católicos y siete judíos. Por último, respecto del dominio del alemán, se señalaba que veintiocho de ellos eran de lengua materna alemana; es decir, que además de los dieciocho señalados como de nacionalidad alemana, había otros diez de lengua materna alemana; a todas luces esos diez restantes habría que buscarlos entre los seis suizos, los tres rusos y los trece chilenos.

En cuanto al colegio alemán de Temuco, en $1909^{28}$ asistían en total setenta y un alumnos, de los cuales seis solamente eran señalados de nacionalidad alemana siendo los sesenta y cinco restantes considerados de nacionalidad chilena. Respecto de la religión, cincuenta y ocho eran designados como protestantes, doce como católicos y uno como judío. En lo que se refiere al dominio del alemán, cincuenta y nueve alumnos eran considerados de lengua materna alemana, por lo que no cabe duda que la mayoría de los sesenta y cinco alumnos considerados de nacionalidad chilena provenían en realidad de hogares de lengua alemana.

Como podemos ver, en 1909 en los colegios de Traiguén y Temuco había una predominancia de la lengua materna alemana y de la confesión protestante entre el alumnado, lo que se repite en los otros nueve colegios de los cuales se tiene información para ese año ${ }^{29}$.

\footnotetext{
${ }^{24}$ Ende, 1910: 67.

25 Ende, 1910: 68

${ }^{26}$ Frey, 1910: 358-359.

27 Frey, 1910: 360-361.

${ }^{28}$ Frey, 1910: 360-361.

29 Frey, 1910: 360-361.
} 
Es posible concluir entonces que la mayor parte del alumnado que frecuentaba los colegios alemanes en el primer decenio del siglo XX provenía de familias de cultura alemana y religión protestante. Esto se evidencia al considerar el porcentaje total de alumnos de religión protestante de estos colegios en 1909, pues de un total de 332 alumnos de los nueve colegios contabilizados, el 81\% (269) eran protestantes; del porcentaje restante, un 16,6\% (55) eran católicos y un 2,4\% (8) eran judíos ${ }^{30}$.

No tenemos mayores antecedentes sobre la declinación y cierre de la mayoría de estos colegios alemanes, pero sin duda que muchas familias de colonos abandonaron, luego de algunos años, sus antiguos lugares de residencia en las colonias rurales, trasladándose junto con sus hijos a los centros urbanos más importantes; por otra parte, la llegada de la escuela pública a gran parte de la región debe haber hecho mella en establecimientos que habían surgido como una alternativa autogestionada frente a la casi ausencia de la instrucción pública.

Al parecer, después de una primera etapa de multiplicación de los proyectos educativos alemanes que va de 1887 a 1906, viene un período de estabilización, que es cuando se sitúa el estudio de Frey (1909), para posteriormente, iniciarse un proceso de declinación y concentración de esfuerzos en un solo gran proyecto educativo que pudiera irradiar a toda la región desde su principal centro urbano, tanto en la educación primaria como secundaria. Al menos esa puede ser una interpretación plausible que explicaría la perennidad e importancia adquirida por el Colegio Alemán de Temuco, el cual constituye en la actualidad uno de los principales establecimiento de educación privada básica y media de toda la región.

\subsection{El Hogar Suizo La Providencia de Traiguén}

Un caso particular lo constituye el llamado "Hogar Suizo La Providencia" situado en un gran predio agrícola próximo a la ciudad de Traiguén, tanto por ser un establecimiento que se mantiene vigente hasta la actualidad con un cierto sello suizo y especializado en la educación agrícola, como por ser un proyecto educativo originado en una misión protestante helvética destinada a ayudar en la difícil situación de algunos colonos suizos y sus descendientes.

Fundado en 1893 por el pastor Arnoldo Leutwyler, el llamado "Asilo de Huérfanos La Providencia" tuvo por función primera hacerse cargo de los hijos de colonos suizos que quedaban huérfanos y ocuparse de la enseñanza de los hijos de colonos en general. Consistía en un orfanato donde funcionaba igualmente una escuela de enseñanza primaria mixta a la que acudían los niños internos y los del sector. A partir de 1897, la obra fue financiada por la "Sociedad Protectora del Huérfano" creada por el pastor Leutwyler.

Se puede decir que el "Hogar La Providencia" tuvo su origen en una misión protestante y mantuvo un vínculo estrecho con Suiza hasta 194931. Desde su génesis,

30 Frey, 1910: 360-361.

31 Hasta esa fecha (1949) esta institución mantiene una relación estrecha con Suiza y con el desarrollo de una educación suizo-germana de orientación protestante, si bien se trata de un colegio laico; el director del establecimiento y los maestros provenían de Suiza. Posteriormente, se produce una desvinculación creciente entre el colegio y los antiguos núcleos de colonos, ampliando su cobertura a toda la región y tomando una 
la comunidad de inmigrantes suizos se vinculó y participó del proyecto; en este sentido, compartió las características que presentaron las escuelas francesas y alemanas de colonias, en tanto proyectos educativos autogestionados y de carácter más bien laico o interconfesional. En todo caso, la enseñanza impartida en el "Hogar La Providencia" estuvo mucho más próxima de aquella impartida en los colegios alemanes que de la impartida en las escuelas francesas, tanto por su orientación protestante como por la presencia del alemán.

\subsection{Los Anglicanos y el Colegio Inglés de Temuco}

Una organización misional ligada a la iglesia anglicana que centró su actividad en América del Sur, la SAM (South American Missionary Society) fue una de las primeras instituciones no católicas que tuvieron un rol importante en el medio educativo de la Araucanía. La SAM inicio sus actividades de evangelización en el litoral de la actual provincia de Arauco, fundando una primera misión en Lota, en 1861, y una segunda en Lebu, en $1867^{32}$.

Entre 1893 y 1890, la SAM inició sus actividades de evangelización y educación en la actual región de la Araucanía creando escuelas en Quino, Chol-Chol y Maquehue Pelal $^{33}$ destinadas a los niños mapuches, pero a la cual también asistían los hijos de colonos de los respectivos sectores.

Según Venezian ${ }^{34}$ la SAM habría fundado igualmente una escuela en Temuco durante este mismo período (1893-1890); sin embargo, no tenemos otros antecedentes y sólo en 1910 aparecen referencias de la creación de dos escuelas primarias anglicanas en Temuco, una de hombres y otra de niñas, claramente orientadas a entregar una educación inglesa a los hijos de inmigrantes y de chilenos ${ }^{35}$.

Una de esas dos escuelas, la de varones, fue dirigida posteriormente por el matrimonio inglés constituido por George Chaytor y Ethel Shilcock, el cual llegó a Temuco a fines de 1914 a petición de la SAM para hacerse cargo de dicha escuela; se fundó así en 1915 el Colegio Inglés de Temuco, el cual sería posteriormente adquirido por el matrimonio y pasaría a ser el actual "Colegio Inglés George Chaytor ${ }^{36}$.

Si bien este colegio no fue fruto de la acción directa de la colonia inglesa, inicialmente estuvo muy asociado a ella, tanto por su orientación religiosa anglicana como por el predominio del inglés como lengua de enseñanza; en los primeros años

orientación técnico-profesional centrada en la formación agrícola media de varones, lo que es facilitado por las características de predio agrícola que posee el recinto donde se sitúa. Desde 1985 se agrega la especialidad forestal a la agrícola al ahora llamado Liceo Agrícola y Forestal Suizo "La Providencia" (Cf.: entrevista a su director Sr. Dufey, 2004 y documentos internos facilitados por él)

32 Venezian, 1993: 23.

33 Venezian, 1993: 269; Bazley, [1999]: 105.

34 Venezian, 1993: 269.

35 Bazley, [1999]: 105.

${ }^{36}$ Hasta 1984, Ethel Shilcock, ya viuda de George Chaytor, continuó a cargo del establecimiento, transfiriendo ese año su administración al Centro de Padres y Apoderados. Se creó entonces la Sociedad Inmobiliaria Nueva Escocia con acciones adquiridas por padres y apoderados; dicha sociedad llevó a cabo la construcción de un nuevo establecimiento y la administración de sus bienes inmobiliarios. A partir de 1990 el colegio amplió su cobertura educativa, agregando a la enseñanza básica, la educación media; en los dos casos se trata de enseñanza mixta (Reseña histórica oficial del Colegio Georges Chaytor, 2006 y Entrevistas a las profesoras Irene Hermosilla y Rhoda Cullen, 2007). 
de funcionamiento, el colegio recibía en su internado a una población promedio de 60 alumnos varones ${ }^{37}$. En todo caso, posteriormente, la presencia de la colonia inglesa se hará más difusa y, al menos en cuanto a la nacionalidad del alumnado, ésta dejará de ser significativa, ya que hacia 1934 la casi totalidad de los alumnos eran definidos como de nacionalidad chilena ${ }^{38}$, aunque ciertamente este no es el único indicador a la hora de determinar la importancia lingüística y cultural de una comunidad de origen inmigratorio ${ }^{39}$.

\section{OTROS PROYECTOS EDUCATIVOS PROTESTANTES POSTERIORES}

A esta primera etapa de surgimiento de escuelas privadas laicas y protestantes, asociadas a núcleos de familias de inmigrantes europeos, instaladas principalmente en propiedades rurales de la Araucanía, sigue la fundación de colegios por misiones protestantes. Aunque estas fundaciones posteriores no son el tema de este artículo, conviene, sin embargo, referirse sucintamente a ellas, para una mejor comprensión de la relación entre inmigración, educación y protestantismo.

A diferencia de las primeras escuelas de colonias, estos colegios protestantes no están dirigidos prioritariamente a los hijos de inmigrantes. Sin embargo, como ocurre que es entre los inmigrantes europeos y sus descendientes, principalmente alemanes, ingleses y suizos, donde se encuentra una gran parte de la población protestante de la Araucanía de los primeros decenios del siglo XX, sin duda se produce una conexión estrecha entre dichos inmigrantes, sus descendientes y estos proyectos educativos protestantes. Se trata, en general, de proyectos más ambiciosos que buscan cubrir diversos sectores de la población, en particular, la clase media urbana y la población indígena.

Estos establecimientos nacen en relación directa con proyectos misionales protestantes provenientes fundamentalmente de Estados Unidos; entre ellos encontramos a las iglesias Adventista, Bautista y Metodista.

Los Adventistas iniciaron tempranamente en el siglo XX su labor educativa en la región, fundando en 1905 la Escuela Filadelfia en una zona rural mapuche, y en 1906 el Colegio Adventista de Chile en Púa para la preparación de misioneros. En 1935 fundaron el Colegio Adventista de Temuco, el cual continúa funcionando en la actualidad; otros colegios adventistas funcionan actualmente en Angol, Trovolhue, Pitrufquén y Villarrica.

Los Bautistas llevaron a cabo su más importante proyecto educativo en Temuco, se trata del Colegio Bautista de Temuco perteneciente a la Unión Bautista de Chile, fundado en 1922 como colegio de niñas con 10 alumnas y dos internas bajo la dirección de la misionera estadounidense Agnes Graham. Dicho colegio contó con el apoyo inicial de la Convención Bautista del Sur de los Estados Unidos ${ }^{40}$. En 1923,

\footnotetext{
37 Martínez y Arriagada, 1976: 41.

38 Martínez y Arriagada, 1976: 41.

39 Según la más antigua profesora del Colegio Georges Chaytor, la Sra. Cullen, hija de inmigrantes asentados en Temuco hacia los años 1930, ya en su niñez Temuco no contaba con una verdadera comunidad británica.

${ }^{40}$ Eddinger, 1992: 38.
} 
el colegio extendió su cobertura de kinder a $2^{\circ}$ año de humanidades y adoptó un carácter mixto $^{41}$. Actualmente, además del Colegio Bautista de Temuco, existen colegios bautistas en Padre Las Casas y en Vilcún.

Los Metodistas comenzaron su labor educativa hacia 1920 en el norte de la región, en las cercanías de Angol, con la Escuela Agrícola "El Vergel”, la cual abrió sus puertas en 1920 bajo la dirección del pastor Ezra Bauman, en el emplazamiento del antiguo fundo y vivero creado por el acaudalado agricultor y banquero Manuel Bunster en 1880 y adquirido por la Misión Metodista en $1919^{42}$. Mucho más tardíamente, en 1949, se fundó en Temuco un Colegio Metodista, el cual es administrado en la actualidad por la Primera Iglesia Metodista de Temuco. Otra área importante de desarrollo del proyecto educativo metodista en la región se encuentra en Nueva Imperial, al igual que El Vergel, se trata de educación agrícola dirigida a los hijos de campesinos chilenos y mapuches. Esta labor se inició durante la década de 1940 y tuvo un gran desarrollo en la década de 1950 cuando el Centro Rural Metodista de la Quinta “Duncan-Magnun" mantenía 28 escuelas primarias, una escuela agrícola, un policlínico y dos cooperativas ${ }^{43}$.

Los colegios adventista, bautista y metodista de la ciudad de Temuco tuvieron, y sin duda tienen en la actualidad, un espacio consolidado en la educación primaria y secundaria privada de esta ciudad, al igual que colegios católicos prestigiosos; esto quizás sea una particularidad de Temuco en el sentido de una temprana diversidad confesional en la educación privada. Por otra parte, dichas iglesias desarrollaron una labor importante en la educación rural, particularmente dirigida a la población mapuche, terreno que compartieron con la iglesia anglicana y sobre todo con la iglesia católica.

\section{CONCLUSIÓN}

Entre los dos últimos decenios del siglo XIX y los dos primeros del siglo XX, se consolidaron las principales comunidades de inmigrantes europeos presentes en la Araucanía, en torno a la ocupación de los espacios rurales que se les asignaron en calidad de colonos. Paralelamente, fueron ocupando los contextos urbanos, ya sea porque se trasladaron a las ciudades, ya sea porque nuevos inmigrantes "libres" se incorporaron a las actividades urbanas, aumentando así la presencia de cada comunidad en dichos contextos.

${ }^{41}$ Eddinger, 1992: 39-40. En la actualidad, el Colegio Bautista de Temuco cuenta con enseñanza mixta básica y media completa reconocida por el Estado y da especial importancia al aprendizaje del inglés, su matrícula era de 1000 alumnos en el año 2005 .

${ }_{42}$ El fundo "El Vergel", propiedad agrícola de 1.500 hectáreas, se convirtió en un importante centro metodista de enseñanza agrícola y de actividad productiva; allí funcionó además de la Escuela Agrícola, una Escuela Vocacional Femenina (Vergara, 1962: 64-65). En la actualidad, funciona sólo la Escuela Agrícola en calidad de Liceo mixto con enseñanza secundaria de primero a cuarto medio. El Vergel posee además un hermoso parque y un museo con piezas arqueológicas y etnográficas de gran valor, que dan cuenta de la actividad científica e intelectual de Dillman S. Bullock, profesor anglicano llegado a Chile desde Estados Unidos en 1902 y convertido al metodismo.

43 Vergara, 1962: 64-65. A partir de los años 1960, la actividad educativa metodista en dicha área comenzó a disminuir, la mayoría de sus escuelas pasaron al sistema educativo público. En la actualidad, la Obra Rural Metodista mantiene en la región tres establecimientos "particulares subvencionados": dos en el área de Nueva Imperial, el Liceo Agrícola Metodista La Granja (1993) y la Escuela Básica Rulo Nº 118 (1945 aprox.) y uno en las cercanías de Loncoche, la Escuela Básica Lolo-Ruka N²2 (Cf. Entrevista a Daniel Martínez, 2006). 
Una buena parte de estos inmigrantes, en particular alemanes, suizos e ingleses, no eran católicos sino protestantes o anglicanos, lo que implicó que en la propia génesis del proceso de ocupación territorial de la Araucanía surgiera la cuestión de la libertad de culto y de la educación privada no católica.

De alguna manera, la escuela fue un terreno propicio en el cual cada comunidad de inmigrantes pensó e implementó estrategias para conservar la identidad de origen o proyectarse al futuro. Dos factores fundamentales justificaron la implementación de proyectos educativos propios: la lengua y la religión.

Esto fue particularmente cierto en los casos de los inmigrantes alemanes y franceses, pues la escuela expresó la concreción de dos proyectos de orientación diferente: uno de lengua alemana y de tradición protestante, y otro de lengua francesa y de tradición católica. La escuela, sin embargo, tuvo más bien un carácter laico o interconfesional abierto a la comunidad, aunque sin duda constituyó un espacio de reconocimiento identitario para los hijos y descendientes de los inmigrantes, identificados lingüística y culturalmente con el proyecto educativo del cual participaban.

\section{PERSONAS ENTREVISTADAS Y CONSULTADAS}

Nuestros más sinceros agradecimientos a las personas abajo señaladas por su valiosa y generosa colaboración.

Sr. Armando Dufey, Director del Liceo Agrícola La Providencia de Traiguén, descendiente de colonos, 2004.

Sr. Armando Lefenda, Habitante de Faja Maisan, descendiente de colonos, historiador local, 2004.

Sr. Juan Salinas, Profesor de Historia y Geografía del Colegio Bautista de Temuco, 2005.

Sr. Daniel Mardones, Pastor de la Sociedad Evangélica de Chile en Temuco, 2005.

Sr. Josef Hund Hund, descendiente de colonos de Pichi Chelle, 2005.

Sr. Armando Fuentes, Pastor de la Iglesia Anglicana de Temuco, 2006.

Sr. Daniel Martínez, Director del Liceo Agrícola Metodista "La Granja”, encargado de la Obra Rural Metodista., 2006.

Sr. Daniel Campos, Pastor de la Iglesia Metodista de Angol, 2006.

Sra. Rhoda Cullen Cox, profesora del Colegio Georges Chaytor, 2007.

Srta. Irene Hermosilla, profesora del Colegio Georges Chaytor, 2007. 
Los colonos y la escuela en la Araucanía: Los inmigrantes europeos y el surgimiento de la educación privada Laica y Protestante en la Región de la Araucanía (1887-1915)

\section{BIBLIOGRAFÍA}

\section{Colecciones}

Anuario Estadístico de la República de Chile (AECh), Oficina Central de Estadísticas, años 1912; 1913; 1914; 1919.

Bulletins de l’Alliance Francaise (B.A.F.), París, años 1884 ( $\left.N^{\circ} 1\right)$ a 1906 ( $\left.N^{\circ} 105\right)$.

Censos Generales de Población de Chile, años 1865 y 1907.

Memorias Anuales del Ministerio de Relaciones Exteriores de Chile (M.RREE), años 1883; 1884; 1888; 1890; 1892; 1894; 1900; 1901; 1908; 1909; 1911; 1912.

\section{Bibliografía}

Aylwin, J. Estudio sobre tierras indígenas de la Araucanía: antecedentes históricolegislativos (1850-1920), Universidad de La Frontera, Temuco, 1995.

Bazley, B. Somos Anglicanos.-: Iglesia Anglicana, Temuco, [1999].

Cohen, J. Los judíos en Temuco, RIL Editores, Santiago, 2002.

Dufey, A. La emigración suiza en la Araucanía (Chile), Impresos Regional Ltda., Victoria, 2000.

Eddinger, R. Historia del Colegio Bautista de Temuco 1922-1992, Colegio Bautista, Temuco, 1992.

Ende, P. Los colonos brandeburgueses en la Frontera, en: Sociedad Científica Alemana de Santiago, Los Alemanes en Chile, Tomo 1, Editorial Universitaria, Santiago, pp. 63-70, 1910.

Frey, J. Los colegios alemanes en Chile, en: Sociedad Científica Alemana de Santiago, Los Alemanes en Chile, Tomo 1, Editorial Universitaria, Santiago, 1910, pp.353-363

Krebs Kaulen, A; Tapia Guerrero, Sor Ú.; Schmid Anwandter, P. Los alemanes y la comunidad chileno-alemana en la historia de Chile, Liga Chileno-Alemana, Santiago, 2001.

Krebs de Wagner, L. 100 años en la Frontera 1885-1985. Homenaje al centenario de la colonización alemana en la Frontera, Comité Coordinador de las instituciones alemanas, Temuco, 1985. 
Instituto Nacional de Estadística. Historia de la División Político-Administrativa 1810-2000, INE, Santiago, 2001.

Martínez, M.; Arriagada, Z. Antecedentes para una historia de la educación en Temuco, Seminario para optar al título de profesor de Estado en Artes Plásticas, Universidad de Chile, Sede Temuco, 1976.

Martínez, C. “Políticas colonizadoras de Chile en el siglo XIX: La ilusión modernizadora, Araucanía 1813-1913", Estudios Sociales, N 69, 1991, pp.79-91.

Radtke, E. 60 Años de Trabajo Misionero Evangélico Alemán en Chile, Sociedad Evangélica de Chile, Santiago, 1993.

Rebolledo, A. La integración de los inmigrantes árabes en la vida nacional: Los sirios en Santiago, Tesis licenciatura en Historia, PUC, Santiago, 1991.

Revista Centenario, Corporación Alianza Francesa de Traiguén, Traiguén, 1991.

Serrano, S. "De escuelas indígenas sin pueblos a pueblos sin escuelas indígenas: La educación en la Araucanía en el siglo XIX", Revista Historia, Vol. 29., 19951996: 423-474.

Venezian, S. Misioneros y maestros: la educación inglesa y norteamericana en Chile en el siglo XIX, Tesis licenciatura en Historia, PUC, Santiago, 1993.

Vergara, I. El protestantismo en Chile, Editorial del Pacífico, Santiago, 1962.

Vernoiry, G. Diez años en Araucanía 1889-1899, Pehuen Editores, Santiago, 2001. 